\title{
Antimicrobial susceptibility and molecular epidemiology of extended-spectrum beta- lactamase-producing Enterobacteriaceae from intensive care units at Hamad Medical Corporation, Qatar
}

\author{
Mazen A Sid Ahmed ${ }^{1 \dagger}$, Devendra Bansal ${ }^{2^{* \dagger}}$, Anushree Acharya ${ }^{2}$, Asha A. Elmi ${ }^{2}$, Jemal M Hamid ${ }^{1}$, \\ Abuelhassan M Sid Ahmed', Prem Chandra', Emad Ibrahim', Ali A Sultan², Sanjay Doiphode', \\ Naser Eldin Bilal ${ }^{3}$ and Anand Deshmukh ${ }^{1 *}$
}

\begin{abstract}
Background: The emergence of extended-spectrum beta-lactamase (ESBL)-producing isolates has important clinical and therapeutic implications. High prevalence of ESBL-producing Enterobacteriaceae has been reported in the literature for clinical samples from a variety of infection sites. The present study was undertaken to evaluate the prevalence of ESBL-producing Enterobacteriaceae, and to perform molecular characterization and antimicrobial susceptibility testing of clinical isolates from patients admitted to the intensive care units at Hamad Medical Corporation, Doha, Qatar, from November 2012 to October 2013.

Methods: A total of 629 Enterobacteriaceae isolates were included in the study. Identification and susceptibility testing was performed using Phoenix (Becton Dickinson) and the ESBL producers were confirmed by double-disk potentiation as recommended by the Clinical and Laboratory Standards Institute. Molecular analysis of the ESBL producers was performed by polymerase chain reaction.

Results: In total, 109 isolates (17.3 \%) were confirmed as ESBL producers and all were sensitive to meropenem in routine susceptibility assays. Most of the ESBL producers (99.1\%) were resistant to amoxicillin/clavulanic acid and ceftriaxone and $93.6 \%$ were resistant to cefepime. Among the ESBL-producing genes, bla $a_{\mathrm{CTX}-\mathrm{M}}(66.1 \%)$ was the most prevalent, followed by bla $a_{\mathrm{SHV}}(53.2 \%)$ and bla

Conclusions: These findings show the high prevalence of ESBL-producing Enterobacteriaceae within the intensive care units at Hamad Medical Corporation, Qatar, and emphasize the need for judicious use of antibiotics and the implementation of strict infection control measures.
\end{abstract}

Keywords: Gram-negative bacteria, Extended-spectrum beta-lactamase, Antimicrobial Susceptibility, Molecular epidemiology, Qatar

\footnotetext{
* Correspondence: deb2022@qatar-med.cornell.edu; adeshmukh@hamad.qa

${ }^{\dagger}$ Equal contributors

${ }^{2}$ Department of Microbiology and Immunology, Weill Cornell Medicine-Qatar,

Doha, Qatar

'Division of Clinical Microbiology, Department of Laboratory Medicine and Pathology, Microbiology Section, Hamad Medical Corporation, Doha, Qatar Full list of author information is available at the end of the article
} 


\section{Background}

The emergence of extended-spectrum beta-lactamase (ESBL)-producing Enterobacteriaceae, particularly Escherichia coli and Klebsiella pneumoniae, presents a significant threat to human health and these organisms are now listed among the six drug-resistant pathogens for which there are few potentially effective drugs [1]. The first case of ESBL was reported in the 1980s in Europe and subsequently in the United States, soon after the introduction of third-generation cephalosporins [2]. Over the last two decades, there has been an exponential increase worldwide in $\beta$-lactamase production and the prevalence of ESBL-producing Enterobacteriaceae, contributing to a significant increase in antimicrobial resistance [3-5]. Importantly, infections with ESBL-producing pathogens are associated with poor clinical outcomes, longer hospital stays, higher mortality rates, and greater hospital expenses $[6,7]$. In addition, there has been a rapid and widespread dissemination of ESBL-producing Enterobacteriaceae in communities as well as in hospital-associated infections, resulting in a worldwide health crisis [8].

The ESBL genes are predominantly plasmid encoded [9] and most belong to the class A $\beta$-lactamases, which can be divided into three genotypes: TEM, SHV, and CTX-M [6]. During the 1990s, ESBL-producing Enterobacteriaceae, E. coli and K. pneumonia, were described mainly as members of the TEM- and SHV- $\beta$-lactamase families [10]. Later in 2000, K. pneumoniae was reported as the major ESBL producer with TEM and SHV as the predominant genotypes [3]. However, ESBL-producing E. coli of the CTX-M genotype is now more prevalent in Western and Asian countries [3]. The CTX-M $\beta$ lactamases comprise more than 50 different types, which can be divided into five groups based on their amino acid identities: CTX-M-1, CTX-M-2, CTX-M-8, CTX-M-9, and CTX-M-25 [11, 12].

Several reports have described the prevalence of ESBLs in the Middle East North Africa (MENA) region and most of the Gulf Cooperation Countries [13]. However, there is insufficient scientific data on the epidemiology of ESBLs available from the State of Qatar. To the best of our knowledge, this is the first study on the molecular epidemiology and antimicrobial susceptibilities of ESBL-producing Enterobacteriaceae from patients in the State of Qatar.

\section{Methods}

\section{Study design}

The present prospective study was conducted on routine specimens received at the Microbiology Laboratory of the Department of Laboratory Medicine and Pathology, Hamad Medical Corporation (HMC), from patients admitted to the intensive care units (ICUs) at HMC. This study was approved (Protocol no. RC/75813/2013) by the Institutional Review Board of HMC.

\section{Clinical isolates/strains}

A total of 629 Enterobacteriaceae isolates (a single isolate per patient) were collected between November 2012 and October 2013 from various clinical specimens as part of routine clinical care. These clinical isolates were preserved at $-70{ }^{\circ} \mathrm{C}$ for further analysis. For each individual, age, nationality, and clinical history were collected from patients' medical records. Infections occurring more than $48 \mathrm{~h}$ after admission were considered hospital acquired [14].

The standard strains of E. coli (ATCC 25922) and K. pneumoniae (ATCC 700603) were used for identification and antimicrobial drug susceptibility testing and $K$. pneumoniae (NCTC 13368), E. coli (NCTC 13351), and E. coli NCTC (13353) were used as positive controls for bla $a_{\mathrm{SHV}}, b l a_{\mathrm{TEM}}$, and $b l a_{\mathrm{CTX}-\mathrm{M}}$ in polymerase chain reaction (PCR) assays, respectively.

Identification and antimicrobial drug susceptibility testing The identification and antimicrobial susceptibility test was performed with Phoenix using the NMIC/ID-5 panel according to the manufacturer's recommendations (BD Biosciences, Heidelberg, Germany). All samples, which tested positive for ESBL by Phoenix or showed an MIC of $>2 \mu \mathrm{g} /$ $\mathrm{mL}$ for ceftazidime, aztreonam, and ceftriaxone, were consequently confirmed by a double-disk potentiation test with ceftazidime, amoxicillin/clavulanic acid, ceftriaxone, and cefoxitin antibiotics and interpretation was carried out as previously described $[15,16]$. Briefly, a microbial suspension with 0.5 McFarland turbidity was inoculated onto Mueller-Hinton agar (Oxoid Ltd., Basingstoke, Hampshire, England), followed by overnight incubation at $37{ }^{\circ} \mathrm{C}$, and susceptibility/resistance patterns were determined.

\section{DNA extraction and detection of ESBL genes by PCR}

Bacterial DNA was extracted using the boiling lysis method [17]. Briefly, a few colonies were suspended in Tris-EDTA buffer (pH 8.0). The suspensions were boiled at $100{ }^{\circ} \mathrm{C}$ for $10 \mathrm{~min}$ and subsequently centrifuged at $15871 \mathrm{~g}$ for $5 \mathrm{~min}$. The supernatant was transferred to new tubes and stored at $-20{ }^{\circ} \mathrm{C}$ for subsequent PCR analysis. PCR reactions were carried out using the following protocol: $2 \mu \mathrm{L}$ of extracted DNA was combined with $12.5 \mu \mathrm{L}$ of $2 \mathrm{X}$ master mix, $1 \mu \mathrm{L}$ forward and $1 \mu \mathrm{L}$ reverse primer, and the mixture was made up to a $25 \mu \mathrm{L}$ volume with nuclease-free water. PCR amplification (Veriti 96 Well Thermal Cycler-Applied Biosystems, Pittsburg, PA, USA) was initiated at $96{ }^{\circ} \mathrm{C}$ for 1 min, followed by 35 cycles at $96{ }^{\circ} \mathrm{C}$ for $1 \mathrm{~min}$, annealing $\left(55{ }^{\circ} \mathrm{C}\right.$ for TEM, and $50{ }^{\circ} \mathrm{C}$ for SHV and CTX-M-1) for 1 min, and extension at $72{ }^{\circ} \mathrm{C}$ for $2 \mathrm{~min}$. Final extension was at $72{ }^{\circ} \mathrm{C}$ for $10 \mathrm{~min}$. All samples were analyzed in duplicate. A positive control (plasmid carrying cloned ESBL gene fragment) and a negative control (nuclease- 
free water) were included in each amplification reaction. After the last cycle, the products were stored at $4{ }^{\circ} \mathrm{C}$ for further analysis. The PCR products (1/10 volume) were analyzed by gel electrophoresis

(Bio-Rad, Hercules, CA, USA) using $2 \%$ agarose gels in $1 \mathrm{X}$ TAE buffer (Tris-acetate EDTA). The gels were stained with ethidium bromide (Sigma, St. Louis, MO, USA), and the PCR products were visualized under ultraviolet light. A single band with an amplicon size of $1,150 \mathrm{bp}$ was observed for TEM, $885 \mathrm{bp}$ for SHV, and $499 \mathrm{bp}$ for the CTX-M-1 group (Table 1) [18].

\section{Results}

\section{Demographic characteristics of the study population}

Samples were collected from patients with suspected bacterial infection from different ICUs $(29.4 \%$ from Medical, $28.5 \%$ Surgical, $16.5 \%$ Trauma, $15.6 \%$ Pediatric, and $10 \%$ from Neonatal) attached to HMC hospitals. Samples were subjected to culturing and subsequent pathogen identification. The demographic profile of the studied population is summarized in Table 2 and shows that $59.6 \%(n=65)$ of samples were collected from male patients and $40.4 \%(n=44)$ of samples were from female patients. Samples were collected from patients ranging in age from 1 month to 86 years, with a mean age of 40.31 years $(S D=26.79)$. It is noteworthy that $68.8 \%$ of patients were non-Qatari and $86.2 \%$ were admitted to hospital for $>2$ days.

\section{Distribution of clinical isolates}

During the study period, a total of 109 (17.3 \%) ESBL-producing Enterobacteriaceae were isolated. Amongst the isolates, $K$. pneumoniae $(51.4 \%, n=56)$ was the most common ESBL-producing organism, followed by E. coli $(34.7 \%, n=38)$, and the numbers of organisms other than E. coli and $K$. pneumoniae were low (13.8 \%, $n=15)$ (Fig. 1). These pathogens were isolated from a variety of clinical samples, respiratory $35.8 \%(n=39)$, blood $27.5 \%(n=30)$, urine $24.8 \%(n=27)$, fluids $6.4 \%(n=7)$, and others $5.5 \%(n=6)$ (Fig. 1$)$.

\section{Antimicrobial resistance/susceptibility profile of ESBL isolates}

Antibiotic resistance/susceptibility patterns of ESBLproducing pathogens are shown in Fig. 2. Among the carbapenems, all ESBL-producing isolates showed $100 \%$
Table 2 Demographic profile of the study population infected with extended-spectrum beta-lactamase-producing pathogens in the State of Qatar

\begin{tabular}{lllllll}
\hline & Total & \multicolumn{3}{l}{ Nationality } & & \multicolumn{2}{l}{ Hospital stay } \\
Gender $\downarrow$ & No. (\%) & Qatari & non-Qatari & & $<2$ days & $>2$ days \\
\hline Male & $65(59.6)$ & 19 & 46 & 6 & 59 \\
Female & $44(40.4)$ & 15 & 29 & 9 & 35 \\
Total No. (\%) & 109 & $34(31.2)$ & $75(68.8)$ & $15(13.8)$ & $94(86.2)$ \\
Age groups (Years) $\downarrow$ & & & & & \\
$\quad 11$ & $18(16.5)$ & 5 & 13 & 4 & 14 \\
$1-12$ & $8(7.4)$ & 4 & 4 & 2 & 6 \\
$13-30$ & $13(11.9)$ & 2 & 11 & 0 & 13 \\
$31-50$ & $24(22)$ & 3 & 21 & 3 & 21 \\
$>50$ & $46(42.2)$ & 20 & 26 & 6 & 40
\end{tabular}

susceptibility to meropenem and slightly reduced susceptibility to imipenem $(99.1 \%)$ and ertapenem $(97.2 \%)$. Among the $\beta$-lactam/ $\beta$-lactamase inhibitor combinations, $78 \%$ were sensitive to piperacillin/tazobactam, whereas $99.1 \%$ were resistant to amoxicillin/clavulanic acid. Susceptibilities to cephalosporins were found to be very low (ceftriaxone $0.9 \%$ and cefepime $6.4 \%$ ). The clinical isolates showed high sensitivity to amikacin $(97.2 \%)$ compared with gentamicin (67 \%) amongst the aminoglycosides. The susceptibility to other classes of antimicrobials such as tigecycline, ciprofloxacin, and trimethoprim/sulfamethoxazole was relatively low (64.2\%, $60.6 \%$, and $38.5 \%$ respectively).

\section{Molecular genotyping of ESBL-producing isolates}

The ESBL-producing pathogens confirmed by phenotypic methods were also molecularly analyzed. Of the 109 ESBL isolates, 24.7 \% harbored multiple bla genes simultaneously and the prevalence of $b l a_{\text {CTX-M }}$ was highest at $66.1 \%$, followed by bla $a_{\mathrm{SHV}}$ at $53.2 \%$, and $b l a_{\mathrm{TEM}}$ at $40.4 \%$ (Fig. 3). The majority of the CTX-M-1-positive isolates were E. coli $(76.3 \%)$ and K. pneumoniae (75 \%); however, all TEM and SHVpositive isolates were K. pneumoniae (53.6\% and $87.5 \%$ respectively). Furthermore, all three bla genes (TEM, SHV, and CTX-M-1) were detected in $46.4 \%$ of $K$. pneumoniae isolates, while two genes (SHV/CTX-M-1) were present in $17.8 \%$ of $K$. pneumoniae and $2.6 \%$ of $E$. coli isolates, with TEM/CTX-M-1 being present in $18.4 \%$ of E. coli and $7.1 \%$ of $K$. pneumoniae and

Table 1 Primers used for polymerase chain reaction amplification of extended-spectrum beta-lactamase genes (TEM, SHV and CTX-M-1)

\begin{tabular}{llll}
\hline Gene & Primer & Sequence $\left(5^{\prime} \mathbf{3}^{\prime}\right)$ & Amplicon size (bp) \\
\hline TEM & TEM-FTEM-R & TTCTTGAAGACGAAAGGGCACGCTCAGTGGAACGAAAAC & 1,150 \\
SHV & SHV-FSHV-R & CACTCAAGGATGTATTGTGTTAGCGTTGCCAGTGCTCG & 885 \\
CTX-M-1 group & CTXM1-FCTXM1-R & GACGATGTCACTGGCTGAGCAGCCGCCGACGCTAATACA & 499 \\
\hline
\end{tabular}




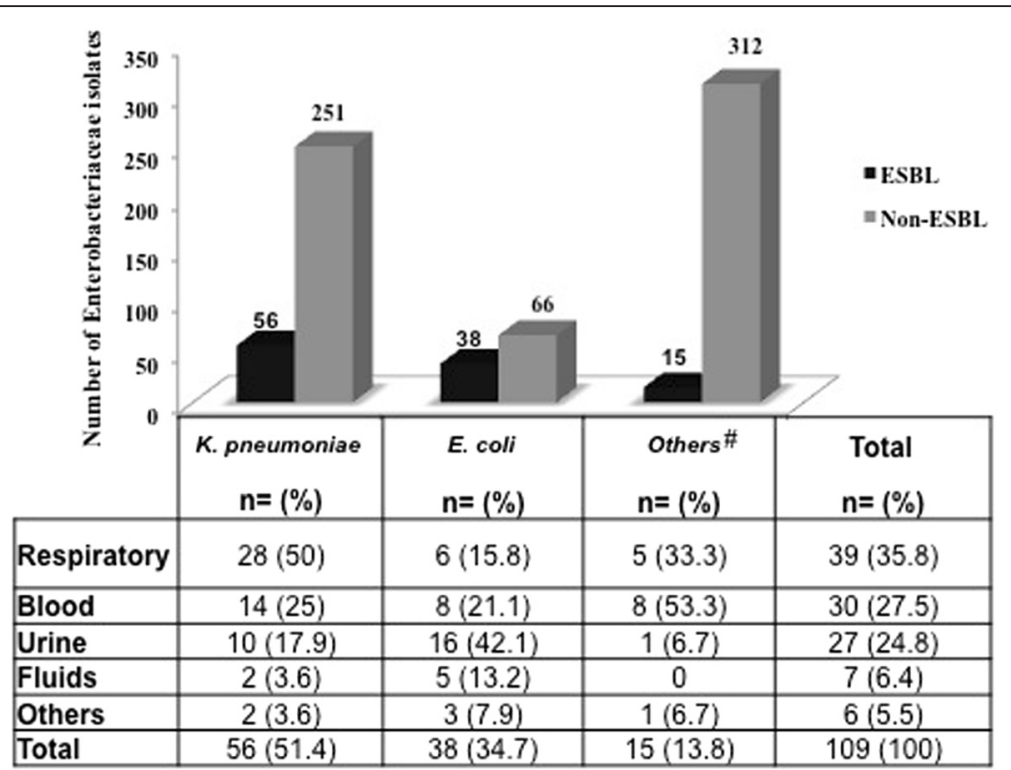

Fig. 1 Distribution of extended-spectrum beta-lactamase-producing organisms among Enterobacteriaceae isolates and the site of isolation. \# Enterobacter cloacae, Citrobacter amalonaticus, Enterobacter aerogenes, Citrobacter amalonaticus, Serratia marcescens, Citrobacter braakii, Citrobacter freundii, Klebsiella oxytoca, Proteus penneri

TEM/SHV being detected in only $5.3 \%$ of $E$. coli isolates (Fig. 3). None of the bla genes were detected in Serratia marcescens, Citrobacter braakii, Citrobacter freundii, Klebsiella oxytoca, or Proteus penneri (data not shown).

\section{Discussion}

In this study, we examined the prevalence of ESBLproducing Enterobacteriaceae and carried out molecular characterization and antimicrobial susceptibility testing

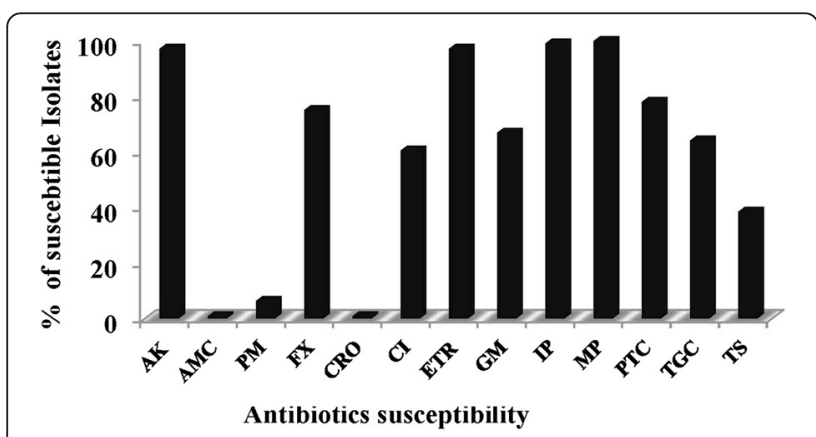

Fig. 2 In vitro activity of the identification and antimicrobial susceptibility test panel of antimicrobial agents against clinical extended-spectrum beta-lactamase-producing isolates. The in vitro activity of antimicrobial agents against clinical isolates was analyzed by BD Phoenix. AK (amikacin), AMC (amoxicillin/ clavulanic acid), PM (cefepime), FX (cefoxitin), CRO (ceftriaxone), Cl (ciprofloxacin), ETR (ertapenem), GM (gentamicin), IP (imipenem), MP (meropenem), PTC (piperacillin/tazobactam), TGC (tigecycline), TS (trimethoprim/sulfamethoxazole) of clinical samples from patients admitted to ICUs at HMC, Doha, Qatar. A total of 629 Enterobacteriaceae isolates were evaluated for the production of ESBL enzymes. In our study, the major ESBL producer was found to be $K$. pneumoniae followed by E. coli and other species, which is in agreement with previous studies $[13,19]$, but overall the prevalence of ESBL-producing pathogens was substantially lower $(17.3 \%)$ than that reported by other studies from neighboring MENA and Asian countries [13, 20-26]. Methodological differences may explain the differences observed in the measured prevalence levels.

In the present study, the respiratory tract was the major source of ESBL-producing isolates, followed by the blood and other sampling sites. Similar findings have been reported for the ICUs of tertiary care hospitals in Mexico and India, where the major source of ESBLproducing isolates were the respiratory tract and blood, respectively $[27,28]$. However, in the Gulf Cooperation Countries region, urine and blood were reported as the major source of ESBL-producing bacteria [13, 26, 29].

The highest level of resistance, in the current study, was observed to be amoxicillin/clavulanic acid, ceftriaxone, and cefepime, while all isolates were susceptible to meropenem, with decreasing levels of susceptibility to imipenem, ertapenem, amikacin, piperacillin/tazobactam, gentamicin, tigecycline, ciprofloxacin, and trimethoprim/ sulfamethoxazole. Recently, Somily et al. [30] reported similar susceptibility rates among E. coli and K. pneumoniae isolates from a tertiary care hospital at Riyadh, Kingdom of Saudi Arabia. However, susceptibility to 


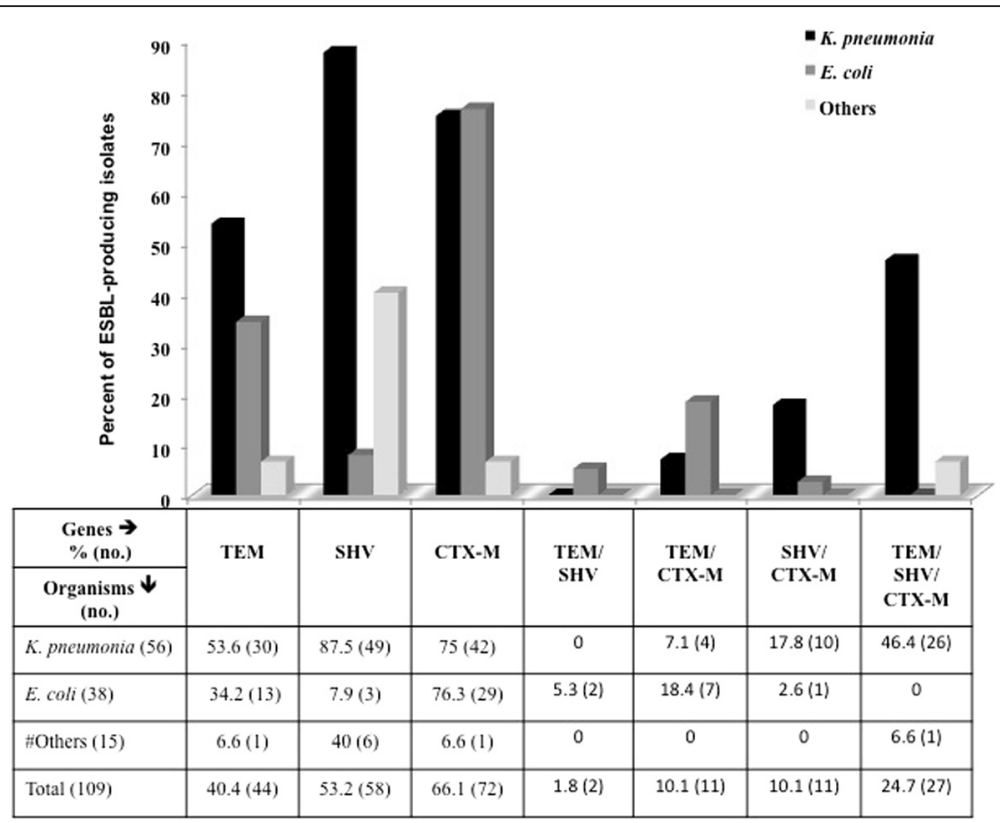

Fig. 3 Distribution of extended-spectrum beta-lactamase-producing genes (TEM, SHV and CTX-M-1) among Enterobacteriaceae isolates in the State of Qatar. \# Enterobacter cloacae, Citrobacter amalonaticus, Enterobacter aerogenes, Citrobacter amalonaticus, Serratia marcescens, Citrobacter braakii, Citrobacter freundii, Klebsiella oxytoca, Proteus penneri

piperacillin/tazobactam and ciprofloxacin were lower in our study compared with isolates from a hospital at Dammam, Kingdom of Saudi Arabia [26]. Furthermore, in Sudan, ESBL-producing E. coli were highly resistant to trimethoprim/sulfamethoxazole and ciprofloxacin but less resistant to amoxicillin/clavulanic acid compared with the present study, but similar susceptibility rates were observed to amikacin and gentamicin [29]. The resistance observed with ertapenem and imipenem compared with merepenem could possibly result from carbapenemase production and/or resistance owing to the loss of porins and/or hyper-production of AmpC; however, this was not further evaluated because of the limitations of the present study.

The predominant genotype of ESBL-producing E. coli and K. pneumonia has changed from TEM and/or SHV to CTX-M-1 [3], and currently, CTX-M has been reported to be the most prevalent genotype among ESBLproducing isolates [13, 26, 28, 31-33]. In this study, molecular genotyping of ESBL-positive isolates showed that the CTX-M-1 gene was the most common among E. coli and $K$. pneumonia followed by SHV and TEM, which was consistent with previous reports from Turkey and India [34, 35]. Additionally, our results showed that only $24.7 \%$ of isolates produced all three genes concurrently and 10.1 $\%$ of isolates co-produced TEM/CTX-M-1 and SHV/ CTX-M-1. These findings obscure the detection rates, affect subsequent treatment strategies, and could be the reason for resistance to $\beta$-lactamase inhibitors [26].

\section{Conclusions}

Compared with other countries in the MENA region, our study shows relatively low prevalence $(17.3 \%)$ of ESBL-producing Enterobacteriaceae. Though lower than in other countries and regions, our study suggests that there is sufficient infection burden to warrant public health interventions. Notably, the majority of isolates were multi-drug resistant and belonged to plasmid-type CTX-M. The emergence of CTX-Mproducing Enterobacteriaceae isolates is of major concern and highlights the need for further surveillance in this area. As meropenem shows good activity against these ESBL producers, it should be restricted for managing patients with suspected Gram-negative bacterial infections with ESBL production. Additionally, antimicrobial stewardship and early detection by active surveillance coupled with an effective infection control program are key to reducing or halting the spread of ESBL producers in hospitals and communities in the State of Qatar.

\section{Compliance with ethical guidelines Ethical standard}

This study was approved by the Research Ethics Committee at HMC, Doha, Qatar.

Competing interests

The authors declare that they have no competing interests. 


\section{Authors' contributions}

MAS, DB, AAS, SD, NB, and AD conceived and designed the study and drafted the manuscript. MAS, DB, AA, AAE, JMH, and AMS performed the experimental work. MAS, DB, PC, and AD analyzed the data. All authors read and approved the final manuscript.

\section{Acknowledgments}

This work was supported by Hamad Medical Corporation (HMC) (12164/12), but this organization played no role in the study design, data collection and analysis, the decision to publish, or preparation of the manuscript. Its contents are solely the responsibility of the authors and do not necessarily represent the official views of the HMC. The authors would like to thank Mr. Muthana A Sid Ahmed for helping in samples collection and Dr. Saad J. Taj-Aldeen for his insightful thoughts and suggestions.

\section{Author details}

'Division of Clinical Microbiology, Department of Laboratory Medicine and Pathology, Microbiology Section, Hamad Medical Corporation, Doha, Qatar. ${ }^{2}$ Department of Microbiology and Immunology, Weill Cornell Medicine-Qatar, Doha, Qatar. ${ }^{3}$ College of Medical Laboratory Sciences, University of Khartoum, Khartoum, Sudan.

\section{Received: 18 October 2015 Accepted: 3 February 2016} Published online: 09 February 2016

\section{References}

1. Talbot GH, Bradley J, Edwards JE Jr, Gilbert D, Scheld M, Bartlett JG. Bad bugs need drugs: an update on the development pipeline from the Antimicrobial Availability Task Force of the Infectious Diseases Society of America. Clin Infect Dis. 2006:42:657-68.

2. Livermore DM, Hawkey PM. CTX-M: changing the face of ESBLs in the UK. J Antimcrob Chemother. 2005;56:451-4.

3. Cantón R, Coque TM. The CTX-M beta-lactamase pandemic. Curr Opin Microbiol. 2006;9:466-75

4. Yezli S, Shibl AM, Memish ZA. The molecular basis of $\beta$-lactamase production in Gram-negative bacteria from Saudi Arabia. J Med Microbiol. 2015:64:127-36.

5. Brolund A. Overview of ESBL-producing Enterobacteriaceae from a Nordic perspective. Infect Ecol Epidemiol. 2014;1:4.

6. Malloy AM, Campos JM. Extended-spectrum beta-lactamases: a brief clinical update. Pediatr Infect Dis J. 2011;30:1092-3.

7. Giske CG, Monnet DL, Cars O, Carmeli Y. Clinical and economic impact of common multidrug-resistant Gram-negative bacilli. Antimicrob Agents Chemother. 2008:52:813-21.

8. Lewis JS, Herrera M, Wickes B, Patterson JE, Jorgensen JH. First report of the emergence of CTX-M-type extended-spectrum beta-lactamases (ESBLs) as the predominant ESBL isolated in a U.S. health care system. Antimicrob Agents Chemother. 2007;51:4015-21.

9. Nakamura T, Komatsu M, Yamasaki K, Fukuda S, Miyamoto Y, Higuchi T, et al. Epidemiology of Escherichia coli, Klebsiella Species, and Proteus mirabilis strains producing extended-spectrum $\beta$-lactamases from clinical samples in the Kinki region of Japan. Am J Cli Pathol. 2012;137(4):620-6.

10. Bradford PA. Extended-spectrum beta-lactamases in the 21st century: characterization, epidemiology, and detection of this important resistance threat. Clin Microbiol Rev. 2001;14:933-51.

11. Lahlaoui H, Ben Haj Khalifa A, Ben Moussa M. Epidemiology of Enterobacteriaceae producing CTX-M type extended spectrum $\beta$-lactamase (ESBL). Med Mal Infect. 2014;44(9):400-4.

12. Shahid M, Singh A, Sobia F, Rashid M, Malik A, Shukla I, et al. bla(CTX-M), bla(TEM), and bla(SHV) in Enterobacteriaceae from north-Indian tertiary hospital: high occurrence of combination genes. Asian Pac J Trop Med. 2011:4:101-5.

13. Zowawi HM, Balkhy HH, Walsh TR, Paterson DL. B-Lactamase Production in Key Gram-Negative Pathogen Isolates from the Arabian Peninsula. Clin Microbiol Rev. 2013;26(3):361-80

14. Ducel G, J Fabry, L Nicolle.Prevention of hospital acquired infections: a practical guide. Prevention of hospital acquired infections: a practical guide Ed. 2. (2002)

15. Giriyapur RS, Nandihal NW, Krishna BVS, Patil AB, Chandrasekhar MR. Comparison of Disc Diffusion Methods for the Detection of ExtendedSpectrum Beta Lactamase Producing Enterobacteriaceae. J Lab Physicians. 2011;3(1):33-6.
16. CLSI. Performance Standards for Antimicrobial Susceptibility Testing: Twenty-Fourth Informational Supplement. CLSI document M100-S24. Clinical and Laboratory Standards Institute:. Wayne, PA; 2014.

17. Queipo-Ortuno Ml, De Dios CJ, Macias M, Bravo MJ, Morata P. Preparation of Bacterial DNA Template by Boiling and Effect of Immunoglobulin $G$ as an Inhibitor in Real-Time PCR for Serum Samples from Patients with Brucellosis. Clin Vaccine Immunol. 2008;15(2):293-6.

18. Shaheen BW, Nayak R, Foley SL, Kweon O, Deck J, Park M, et al. Molecular characterization of resistance to extended-spectrum cephalosporins in clinical Escherichia coli isolates from companion animals in the United States. Antimicrob Agents Chemother. 2011;55(12):5666-75.

19. Guzmán-Blanco M, Labarca JA, Villegas MV, Gotuzzo E. Extended spectrum B-lactamase producers among nosocomial Enterobacteriaceae in Latin America. Braz J Infect Dis. 2014;18(4):421-33.

20. Feizabadi MM, Delfani S, Raji N, Majnooni A, Aligholi M, Shahcheraghi F, et al. Distribution of bla(TEM), bla(SHV), bla(CTX-M) genes among clinical isolates of Klebsiella pneumoniae at Labbafinejad Hospital, Tehran, Iran. Microb Drug Resist. 2010;16(1):49-53.

21. Hawkey PM. Prevalence and clonality of extended-spectrum $\beta$-lactamases in Asia. Clin Microbiol Infect. 2008;14(1):159-65.

22. Bush K, Fisher JF. Epidemiological expansion, structural studies, and clinical challenges of new $\beta$-lactamases from gram-negative bacteria. Annu Rev Microbiol. 2011;65:455-78.

23. Gur D, Hascelik G, Aydin N, Telli M, Gultekin M, Ogulnc D, et al. Antimicrobial resistance in gram-negative hospital isolates: results of the Turkish HITIT-2 Surveillance Study of 2007. J Chemother. 2009:21 (4):383-9.

24. Alfaresi MS, Elkoush AA, Alshehhi HM, Abdulsalam Al. Molecular characterization and epidemiology of extended-spectrum beta-lactamaseproducing Escherichia coli and Klebsiella pneumoniae isolates in the United Arab Emirates. Med Princ Pract. 2011;20(2):177-80.

25. Mokaddas EM, Abdulla AA, Shati S, Rotimi VO. The technical aspects and clinical significance of detecting extended-spectrum $\beta$-lactamase-producing Enterobacteriaceae at a tertiary-care hospital in Kuwait. J Chemother. 2008;20:445-51.

26. Hassan H, Abdalhamidm B. Molecular characterization of extendedspectrum beta-lactamase producing Enterobacteriaceae in a Saudi Arabian tertiary hospital. J Infect Dev Ctries. 2014;8(3):282-8.

27. Garza-Gonzalez E, Mendoza Ibarra SI, Llaca-Dıaz JM, Gonzalez GM. Molecular characterization and antimicrobial susceptibility of extended-spectrum $\beta$-lactamase producing Enterobacteriaceae isolates at a tertiary care centre in Monterrey, Mexico. J Med Microbiol. 2011;60(1):84-90.

28. Sharma M, Pathak S, Srivastava P. Prevalence and antibiogram of Extended Spectrum $\beta$-Lactamase (ESBL) producing Gram negative bacilli and further molecular characterization of ESBL producing Escherichia coli and Klebsiella spp. J Clin Diagn Res. 2013;7(10):2173-7.

29. Ibrahim ME, Bilal NE, Magzoub MA, Hamid ME. Prevalence of Extendedspectrum $\beta$-Lactamases-producing Escherichia coli from Hospitals in Khartoum State, Sudan. Oman Med J. 2013:28(2):116-20.

30. Somily AM, Habib HA, Absar MM, Arshad MZ, Manneh K, Al Subaie SS, et al. ESBL-producing Escherichia coli and Klebsiella pneumoniae at a tertiary care hospital in Saudi Arabia. J Infect Dev Ctries. 2014;8(9):1129-36.

31. Zhao WH, Hu ZQ. Epidemiology and genetics of CTX-M extended-spectrum B-lactamases in Gram-negative bacteria. Crit Rev Microbiol. 2013;39(1):79-101.

32. D'Andrea MM, Arena F, Pallecchi L, Rossolini GM. CTX-M-type $\beta$-lactamases: a successful story of antibiotic resistance. Int J Med Microbiol. 2013;303(6-7):305-17.

33. Stedt J, Bonnedahl J, Hernandez J, Waldenström J, McMahon BJ, Tolf C, Olsen B, Drobni M. Carriage of CTX-M type extended spectrum $\beta$ lactamases (ESBLs) in gulls across Europe. Acta Vet Scand. 2015;57(1):74

34. Bali EB, Açık L, Sultan N. Phenotypic and molecular characterization of SHV, TEM, CTX-M and extended-spectrum- $\beta$-lactamase produced by Escherichia coli, Acinobacter baumannii and Klebsiella isolates in a Turkish hospital. African J Microbiol Res. 2010;4(8):650-4.

35. Goyal A, Prasad KN, Prasad A, Gupta S, Ghoshal U, Ayyagari A. Extended spectrum $\beta$-lactamases in Escherichia coli \& Klebsiella pneumoniae \& associated risk factors. Indian J Med Res. 2009:129(6):695-700. 